\title{
吡咯并菲啶酮构建方法学研究进展
}

\author{
丛 蔚 $a, b$ 徐进宜 ${ }^{*, a, b}$ 姚和权 ${ }^{*, a, b}$ \\ ( ${ }^{a}$ 中国药科大学天然药物活性组分与药效国家重点实验室 南京 210009) \\ ( ${ }^{b}$ 中国药科大学药学院 南京 210009)
}

\begin{abstract}
摘要 吡咯并菲啶酮母核广泛存在于石蒜科生物碱中, 该系列的生物碱展示出很多有价值的生物活性. 吡咯并菲啶酮 独特的结构吸引了众多学者对其合成进行探索研究. 在过去的几十年中, 很多有价值的构建方法被报道, 其中主要以 过渡金属催化或自由基历程等分子内或分子间偶联反应为主. 对其构建方法学进行了系统地综述.

关键词＼cjkstart吡咯并菲啶酮; 方法学; 偶联反应; 金属催化; 自由基反应
\end{abstract}

\section{Recent Advances on Construction of Pyrrolophenanthridone Skeleton}

\author{
Cong, Wei ${ }^{a, b} \quad \mathrm{Xu}$, Jinyi $^{*, a, b} \quad$ Yao, Hequan*,a,b \\ ( ${ }^{a}$ State Key Laboratory of Natural Medicines (SKLNM), China Pharmaceutical University, Nanjing 210009) \\ ( ${ }^{b}$ Department of Medicinal Chemistry, China Pharmaceutical University, Nanjing 210009)
}

\begin{abstract}
Pyrrolophenanthridone is a common structural motif existing in a number of amaryllidaceae alkaloids, many of which possess a wide range of biological activities. The intriguing structural feature and significant biological activity have attracted much attention from synthetic communities. In the past decades, several excellent synthetic methodologies have been reported, in which intramolecular or intermolecular cross-coupling reaction through transition-metal catalyzed or radical pathway represents the most straightforward method for construction of this skeleton. This review focuses on the construction of pyrrolophenanthridone skeleton and total synthesis of amaryllidaceae alkaloids.
\end{abstract}

Keywords pyrrolophenanthridone; methodology; cross-coupling reaction; transition-metal catalyzed; radical reaction

吡咯并菲啶酮(Pyrrolophenanthridone)是一类具有 多环吲哚结构的母核, 广泛存在于一系列石蒜科 (Amaryllidaceae)生物碱 ${ }^{[1]}$ 中. 从 20 世纪 80 年代初至今, 已发现大约 200 种石蒜科生物碱, 其中数十种含有吡咯 并菲啶酮母核 ${ }^{[2]}$ (图 1). 药理活性评价表明, 吡咯并菲啶 酮生物碱具有多种生物活性 ${ }^{[3]}$. 例如, Hippadine (1) 能可 逆地抑制雄性大鼠的生育能力 ${ }^{[4]}$, 而 Kalbretorine (2)对 肿瘤细胞有较强的抗增殖活性 ${ }^{[2 \mathrm{~d}]}$. 众多有希望的药理 活性报道促使学者们对其进行深入地研究, 但是天然吡 咯并菲啶酮生物碱的来源有限, 因此发展高效、实用的 合成方法学十分必要.

吡咯并菲啶酮母核具有独特的并四芳杂环的平面 大 $\pi$ 体系(图 1): $\mathrm{A}$ 环为苯环, B 环为 6 元内酰胺环, $\mathrm{CD}$
环组成吲哚环. 吡咯并菲啶酮生物碱的结构高度相似, 不同之处在于 $\mathrm{A}$ 环含氧取代基的数目和种类, 以及少数 $\mathrm{CD}$ 环连有取代基. 此外, 很多石蒜科生物碱的 C 环未 芳构化或者 $\mathrm{D}$ 环不为吡咯环, 不属于严格定义的吡咯并 菲啶酮生物碱的范畴; 加之对此类生物碱的合成策略与 吡咯并菲啶酮有很大不同，因此本文不作讨论.

近几十年来，吡咯并菲啶酮新颖的结构和众多的生 物活性吸引了有机合成学者广泛的关注，目前已有多种 合成方法报道. 由于吡咯并菲啶酮的 B 环为 6 元内酰胺 环, 易于构建, 多数报道都是以构建 B 环作为关键步骤; 此外少数学者另辟蹊径, 以构建 $\mathrm{CD}$ 环为关键步骤. 本 文综述了近几十年来吡咯并菲啶酮代表性的构建方法, 包括金属催化和自由基历程等分子内和分子间偶联反

\footnotetext{
*E-mail: hyao@cpu.edu.cn; jinyixu@china.com

Received April 22, 2014; revised May 29, 2014; published online June 16, 2014

Project supported by the National Natural Science Foundation of China (No. 21272276) and the Program for Changjiang Scholars and Innovative Research Team in University (No. IRT1193).

国家自然科学基金(No. 21272276)和长江学者和创新团队发展计划(No. IRT1193)资助项目.
} 


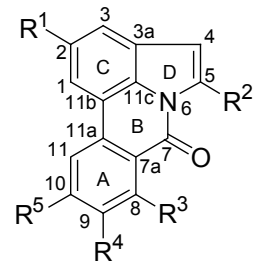

Pyrrolophenanthridone

\begin{tabular}{|c|c|c|c|c|c|c|}
\hline Compd. & Alkaloid & $\mathrm{R}^{1}$ & $\mathrm{R}^{2}$ & $\mathrm{R}^{3}$ & $\mathrm{R}^{4}$ & $\mathrm{R}^{5}$ \\
\hline 1 & Hippadine & $\mathrm{H}$ & $\mathrm{H}$ & $\mathrm{H}$ & \multicolumn{2}{|c|}{$\mathrm{OCH}_{2} \mathrm{O}$} \\
\hline 2 & Kalbretorine & $\mathrm{H}$ & $\mathrm{H}$ & $\mathrm{OH}$ & \multicolumn{2}{|c|}{$\mathrm{OCH}_{2} \mathrm{O}$} \\
\hline 3 & Pratosine & $\mathrm{H}$ & $\mathrm{H}$ & $\mathrm{H}$ & $\mathrm{OMe}$ & $\mathrm{OMe}$ \\
\hline 4 & Pratorimine & $\mathrm{H}$ & $\mathrm{H}$ & $\mathrm{H}$ & $\mathrm{OMe}$ & $\mathrm{OH}$ \\
\hline 5 & Pratorinine & $\mathrm{H}$ & $\mathrm{H}$ & $\mathrm{H}$ & $\mathrm{OH}$ & $\mathrm{OMe}$ \\
\hline 6 & Criasiaticidine A & $\mathrm{H}$ & $\mathrm{H}$ & $\mathrm{H}$ & $\mathrm{OH}$ & $\mathrm{OH}$ \\
\hline 7 & Lycoranine A & $\mathrm{OMe}$ & $\mathrm{H}$ & $\mathrm{H}$ & \multicolumn{2}{|c|}{$\mathrm{OCH}_{2} \mathrm{O}$} \\
\hline 8 & Lycoranine B & $\mathrm{OMe}$ & $\mathrm{Me}$ & $\mathrm{H}$ & \multicolumn{2}{|c|}{$\mathrm{OCH}_{2} \mathrm{O}$} \\
\hline
\end{tabular}

图 1 吡咯并菲啶酮母核以及代表性生物碱

Figure 1 Pyrrolophenanthridone skeleton and representative compounds

应.

\section{1 以构建 B 环为关键步骤}

构建 B 环通常有三种策略: (1)采用分子内偶联, 将 构建 $\mathrm{AC}$ 环之间的 $\mathrm{C}_{11 \mathrm{a}}-\mathrm{C}_{11 \mathrm{~b}}$ 键作为最终环合步骤 (Scheme 1a), 该策略由于吲哚 2 位远比 7 位活泼, 所以 需要采用将 2 位保护或者以吲哚啉为底物等方法避免在 2 位关环; (2)采用分子内偶联, 将构建 $\mathrm{B}$ 环的 $\mathrm{N}_{6}-\mathrm{C}_{7} /$ $\mathrm{C}_{7}-\mathrm{C}_{7 \mathrm{a}}$ 键作为最终环合步骤(Scheme 1b); (3)采用分子 间偶联同时构建 $\mathrm{C}_{11 \mathrm{a}}-\mathrm{C}_{11 \mathrm{~b}}$ 和 $\mathrm{N}_{6}-\mathrm{C}_{7}$ 键 (Scheme 1c). 下 面对上述三种策略进行详细分析.

\section{1 以构建 $\mathrm{C}_{11 \mathrm{a}}-\mathrm{C}_{11 \mathrm{~b}}$ 键作为最终环合步骤}

\subsection{1通过金属催化实现}

自 20 世纪 70 年代以来, 过渡金属催化的交叉偶联 反应已经成为构建碳一碳和碳-杂键的有力手段, 在芳烃 的官能团化反应中广泛应用 ${ }^{[5]}$. 其中钯催化的分子内偶 联已经成为构建多环化合物的有效方法 ${ }^{[6]} .1991$ 年, Grigg 小组 ${ }^{[7]}$ 通过预先将 $\mathrm{AC}$ 环上的偶联位点 $\mathrm{C}_{11 \mathrm{a}}$ 和 $\mathrm{C}_{11 \mathrm{~b}}$ 用碘活化, 再经历钯催化分子内 Stille 偶联完成 Hippadine (1)的合成(Scheme 2a). 随后在 1993 年, Sakamoto 小组 ${ }^{[8]}$ 换用澳活化并采用同样的策略合成 Hippadine (1). 上述方法偶联反应收率都在中等以上, 不需对吲哚 2 位进行保护, 较为便捷. 但该方法存在一 定的缺陷，例如: 需要较多的反应步骤才能完成偶联前 体 9b 的合成(Scheme 2b); 如果要合成吲哚环带有取代 基的天然产物 Lycoranine $\mathrm{B}(8)$ 或者需要对其它天然产 物进行优化改造, 就需要预先合成各种取代的 1,3-二溴
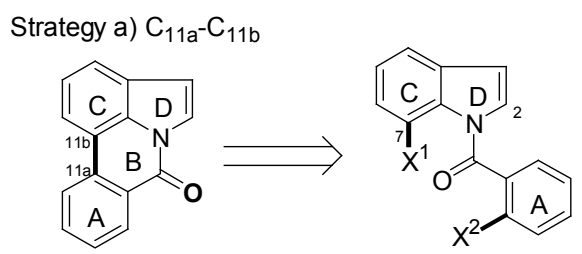

Strategy b) $\mathrm{N}_{6}-\mathrm{C}_{7} / \mathrm{C}_{7}-\mathrm{C}_{7 \mathrm{a}}$<smiles>[Y2]c1ccccc1-c1cccc2ccn([Y1])c12</smiles>

Strategy c) $\mathrm{C}_{11 \mathrm{a}}-\mathrm{C}_{11 \mathrm{~b}}$ and $\mathrm{N}_{6}-\mathrm{C}_{7}$

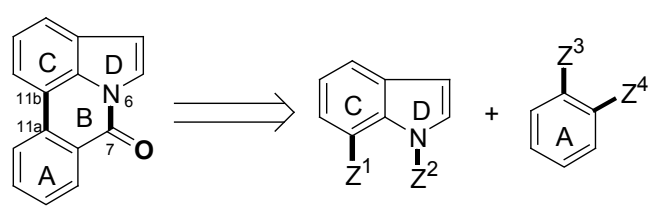

Scheme 1

苯胺 10 和烯烃 11, 这无疑使得合成难度大大增加; 更 重要的是, 该反应需要用到大量的有机锡试剂, 会产生 很多不利于环保的废弃物, 而且预先活化两个位点也使 该反应的原子经济性大大降低.

a)

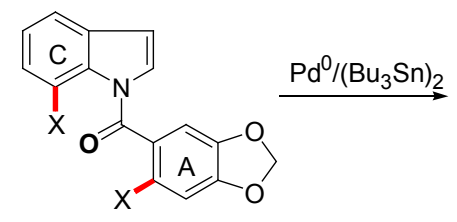

Grigg group: $X=\mid 9 a$

Sakamoto group: $\mathrm{X}=\mathrm{Br} \mathbf{9 b}$

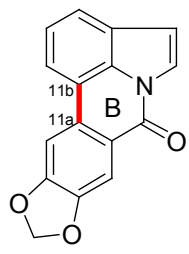

$(60 \%$ for $9 a ; 68 \%$ for $9 \mathbf{b})$

b)

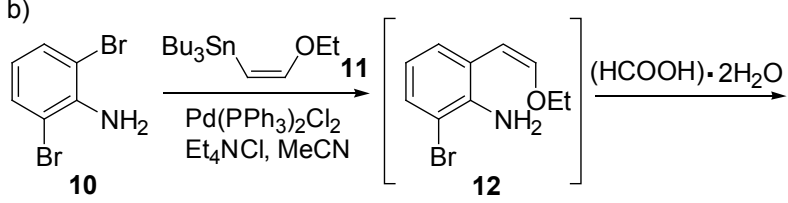<smiles>COc1cc2c(cc1Br)OCO2</smiles>

Scheme 2

2003 年, Knölker 小组 ${ }^{[9]}$ 以二氢吲哚啉衍生物 15 为 起始原料, 通过分子内偶联得到二氢吡咯并菲啶酮生物 碱 Anhydrolycorinone (16), 经芳构化得到 Hippadine (1, Scheme 3). 二氢吲哚啉 2 位的 $\mathrm{C}\left(\mathrm{sp}^{3}\right)-\mathrm{H}$ 难以活化, 因 此不存在反应位点选择性的问题, 免去了额外的保护步 骤; 偶联反应非常迅速，并且同时完成 A 环芳构化和 B 
环芐位氧化, 具有很强的创新性. 但是该方法具有较多 缺陷: 合成偶联前体 15 需要较多的步骤和苛刻的反应 条件; 可能是由于吲哚啉衍生物很不稳定, 关键的偶联 步骤收率较低 (29\%), 并且需要添加等物质的量的 $\operatorname{Pd}\left(\mathrm{PPh}_{3}\right)_{4}$, 因此反应条件还需进一步的优化.
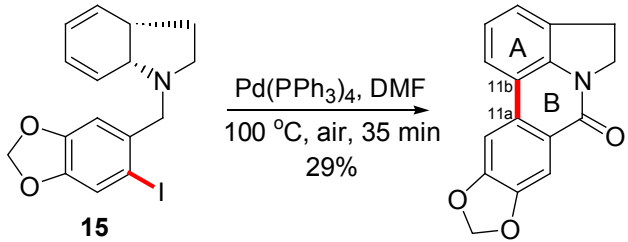

Anhydrolycorinone (16)

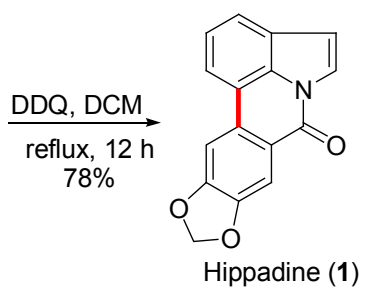

Scheme 3

静红(17)是一种常用的医药、染料中间体, 可以很 容易地还原为吲哚 ${ }^{[10]} .2004$ 年, Garden 小组 ${ }^{[11]}$ 以静红 17 为起始原料, 经过 $N$-芐基化和缩酮保护, 得到偶联前体 20, 在钯催化下能以几乎定量的收率得到环合产物 21, 脱去缩酮保护得到 22 (Scheme 4a). Garden 小组发现, 22 在经历 $\mathrm{BH}_{3}$ 还原时存在反应不完全的问题, 以 $7: 3$ 的 比例生成 23 和 24 的混合物. 虽然 23 能以中等收率还原 为 24, 但是 24 极度不稳定, 在空气中即可发生氧化分 解. 为了解决上述问题, Garden 小组尝试从 22 起始经过 连续还原一氧化反应(Scheme $4 \mathrm{~b})$. 当 22 经历 $\mathrm{BH}_{3}$ 还原 (conditions a)、 $\mathrm{NaCNBH}_{3}$ 还原(conditions b)、Oxone 氧 化后, 得到一种无色的无定型固体, 可能是中间体 $\mathbf{2 4}$ 的盐 25 (结构未经确证), 继续采用碱性 $\mathrm{KMnO}_{4}$ 氧化苄 位(conditions d) 可得到天然产物 Anhydrolycorinone (16). 事实证明, Oxone 氧化的步骤是多余的, 22 经历上述条 件 $a 、 b 、 d$ 就能以 $75 \%$ 的收率得到 16, 随后可以在 $\mathrm{MnO}_{2}$ $\mathrm{SiO}_{2}$ (conditions e) 作用下芳构化得到天然产物 Hippadine (1). 值得一提的是, 除芳构化之外, $\mathrm{MnO}_{2}-\mathrm{SiO}_{2}$ 也可以用作氧化茮位, 仅采用条件 $\mathrm{a} 、 \mathrm{e}$ 能以 $35 \%$ 的收率得到 Hippadine (1). 除了合成 Hippadine (1) 外，该方法也被用作 Pratosine (3)的合成.

Garden 小组首次采用静红作为 “伪装” 的吲哚参与 反应，从而解决了反应位点选择性的问题; 而且关键的 钯催化偶联步骤收率很高, 使得合成效率大大提高; 最 后针对静红部分的还原方法进行了深入地研究, 探索出 一条捷径, 虽然收率有待提高, 但中间体 22 仅通过两步

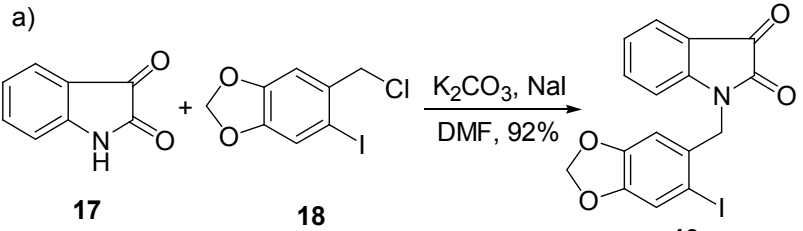

19

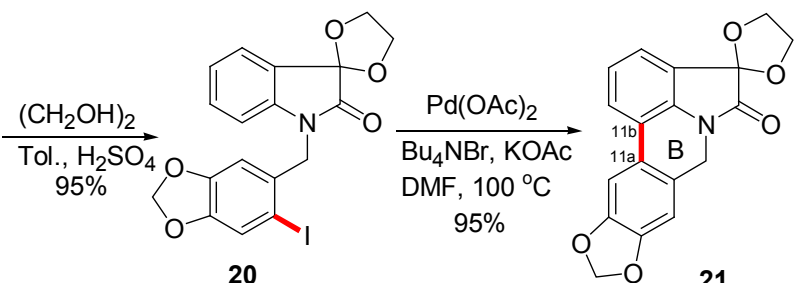

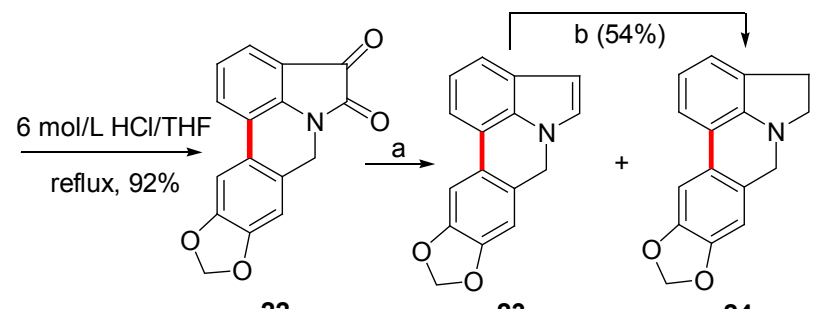

22

23

24

b)

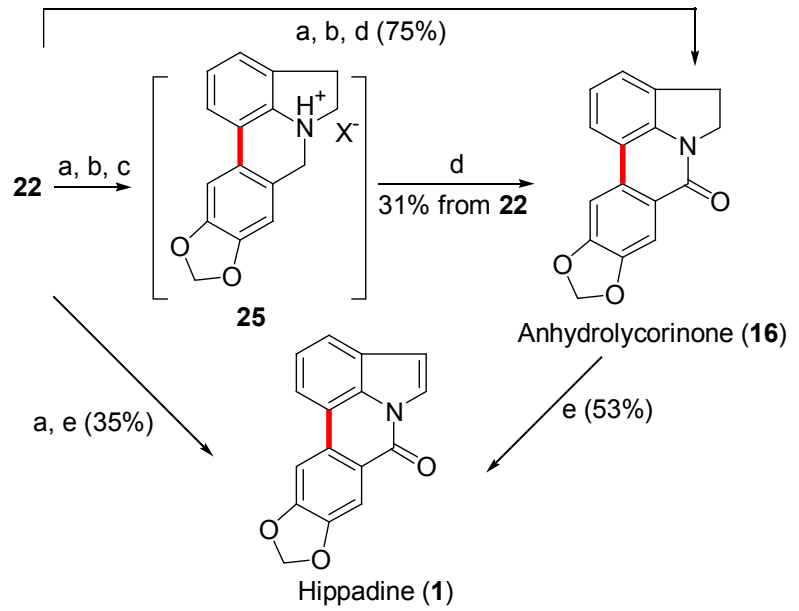

Reaction conditions: (a) $\mathrm{BH}_{3} \cdot \mathrm{HF}$; (b) $\mathrm{NaCNBH}_{3}, \mathrm{AcOH}$; (c) Oxone, $\mathrm{NaHCO}_{3}, \mathrm{H}_{2} \mathrm{O}$ /acetone; (d) $\mathrm{KMnO}_{4}, \mathrm{NaOH}, \mathrm{CH}_{2} \mathrm{Cl}_{2}$; (e) $\mathrm{MnO}_{2}-\mathrm{SiO}_{2}$, benzene, reflux

\section{Scheme 4}

简单地操作就得到终产物也是值得肯定的. 总之，该方 法学路线较为简捷，总收率相对较高，是一种合成吡咯 并菲啶酮生物碱的良好办法.

上述 Grigg 小组 ${ }^{[7]}$ 和 Knölker 小组 ${ }^{[9]}$ 的方法都存在底 物难以制备和修饰的缺陷，不利于大量合成吡咯并菲啶 酮衍生物. 为了改进上述缺陷，达到从简单底物起始以 合成吡咯并菲啶酮的目的，很多学者做了大量的研究工 作. 1999 年, Miki 小组 ${ }^{[12]}$ 报道通过钯催化吲哚衍生物分 子内 Heck 反应来构建吡咯并菲啶酮(Scheme 5). Miki 小组将弜哚 2 位用酯基封闭; 将吲哚 1 位用 2 -溴苄基取 代得到偶联前体 26a/26b, 可以在钯催化下在 7 位发生 
分子内偶联, 以几乎定量的收率得到 $27 \mathbf{a} / 27 \mathbf{b}$. 而后续 酯基的脱除却异常繁琐：先将酯基还原为羟甲基，再经 Dess-Martin 氧化为醛基, 继续用 $\mathrm{MnO}_{2}$ 氧化芐位的同时 将醛基氧化为羧基, 最终在 $\mathrm{RhCl}\left(\mathrm{PPh}_{3}\right)_{3}$ 催化下完成脱 羧. 虽然酯基脱除方法还有待优化, 但上述方法可以从 简单易得的吲哚衍生物起始, 合成天然产物 Pratosine (3)和 Hippadine (1), 因此便于后续天然产物的结构修 饰.

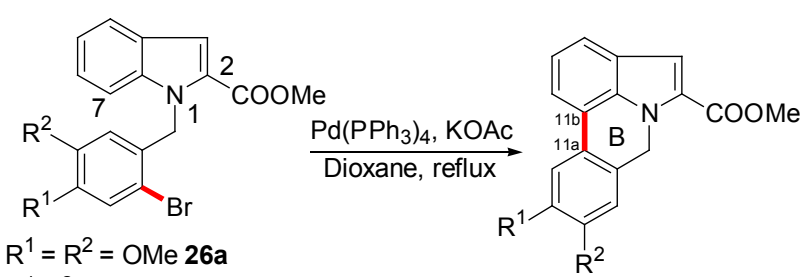
$\mathrm{R}^{1} / \mathrm{R}^{2}=\mathrm{OCH}_{2} \mathrm{O} \mathbf{2 6 b}$

$\mathrm{R}^{1}=\mathrm{R}^{2}=$ OM 27a (96\%) $\mathrm{R}^{1} / \mathrm{R}^{2}=\mathrm{OCH}_{2} \mathrm{O} 27 \mathbf{b}(91 \%)$

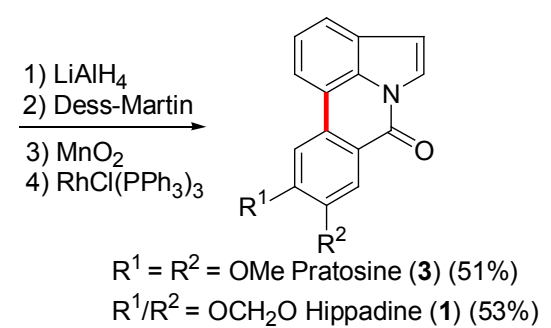

Scheme 5

鉴于上述酯基脱除步骤过于繁琐, 2011 年, Miki 小 组 ${ }^{[13]}$ 对其进行改进(Scheme 6). 用氢氧化锂将甲酯 28 水 解后, 再使用氧化铜一三氧化二铬混合氧化剂, 将羧酸 脱除的同时将芐位氧化得到天然产物 Pratosine (3) 和 Hippadine (1). 遗憾的是, 虽然路线相对简捷, 但最后 一步收率较低, 对总收率的影响较大.<smiles>[R]c1cc2c(cc1[R])-c1cccc3c(C(C)=O)c(C(=O)OC)n(c13)C2</smiles><smiles>[R]c1cc2c3cccc4c(C(=O)O)c(C(=O)O)n(c2cc1[R])c43</smiles>

$R^{1}=R^{2}=$ OMe 29a (99\%) $\mathrm{R}^{1} / \mathrm{R}^{2}=\mathrm{OCH}_{2} \mathrm{O} 29 \mathbf{b}(85 \%)$

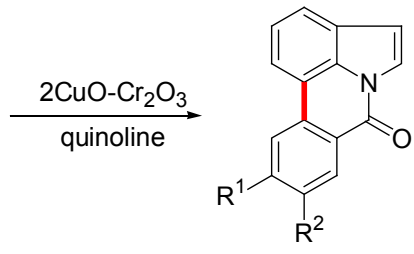

$\mathrm{R}^{1}=\mathrm{R}^{2}=$ OMe Pratosine (3) (20\%)

$\mathrm{R}^{1} / \mathrm{R}^{2}=\mathrm{OCH}_{2} \mathrm{O}$ Hippadine (1) (13\%)
2012 年, Miki 小组 ${ }^{[14]}$ 采用相似方法合成 Kalbretorine (2) 时，继续对中间体 30 的酯基的脱除进行了改进 (Scheme 7). 先将甲酯 30 水解成羧酸 31 , 再通过 $\mathrm{PhI}(\mathrm{OAc})_{2}$ 介导的碘代和氯化亚锡脱碘将羧酸脱除. 该 方法脱除酯基收率很高，操作也相对简捷，比之前报道 的方法有很大改进, 是合成吡咯并菲啶酮生物碱的有效 方法.

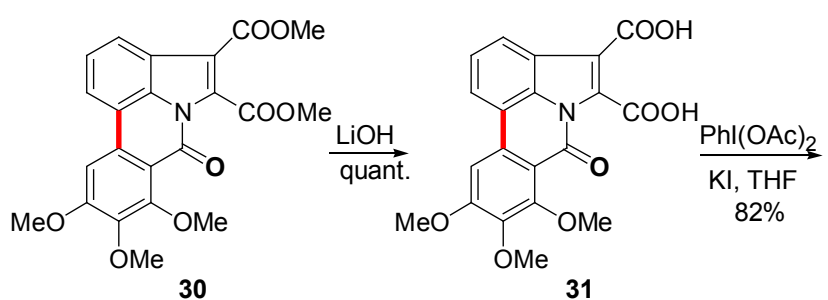

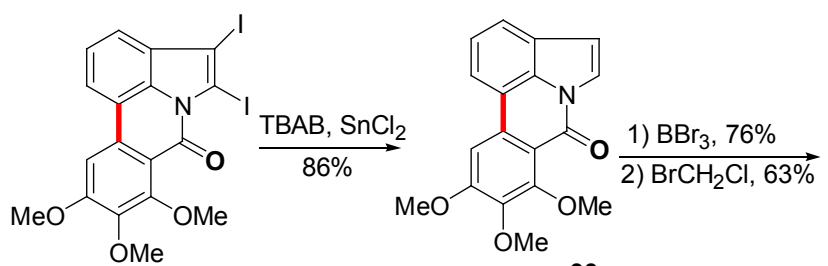
32 33<smiles>O=c1c2c(O)c3c(cc2c2cccc4ccn1c42)OCO3</smiles>

\section{Scheme 7}

最近，我们课题组 ${ }^{[15]}$ 报道了以 1-(2-溴苄基)取代的 吲哚衍生物 34 为原料, 通过钯催化吲哚分子内偶联反 应，一步实现偶联和氧化反应，快速、便捷地构建取代 吡咯并菲啶酮 35 (Scheme 8a). 在底物适应性研究中发 现, 连有各种取代基的 21 个底物均能以中等到良好的 收率得到目标产物. 我们针对该反应的机理进行了探 索, 发现反应是按照先偶联后氧化的步骤进行的, 而且 偶联反应是决速步骤. 继而后续通过同位素示踪法证 实, 产物中羰基氧原子来源于水(Scheme $8 \mathrm{~b})^{[15,16]}$. 该方 法学可用于大量制备各种取代狮并异喹啉酮, 为今后此 类骨架的生物活性研究提供了基础. 但是该方法学也有 局限性: 不适用于合成吲哚环连有吸电子基的目标物 (35i); 也不能直接用于合成 Hippadine (1)等母核 4,5 位 无取代基的天然产物, 因此与 Miki 小组的工作 ${ }^{[12 \sim 14]}$ 有 互补之处.

\subsection{2 通过自由基反应实现}

过渡金属催化反应需要用到昂贵的催化剂, 而且可 能带来重金属残留的问题. 自由基反应 ${ }^{[17]}$ 也是构建联 芳基分子的有效手段，同时可以避免上述缺陷，因此众

Scheme 6 
a) $\mathrm{R}$<smiles>[R1]c1ccc(Cn2c(F)c(P)c3cc([R])ccc32)c(Br)c1</smiles>

34

Representative examples:<smiles>Cc1c(C)n2c(=O)c3ccccc3c3cccc1c32</smiles>

35a $(92 \%)$<smiles>CCc1c(C)n2c(=O)c3ccccc3c3cccc1c32</smiles>

35d $(85 \%)$<smiles>O=c1c2ccccc2c2cccc3c4ccccc4n1c23</smiles>

35g (74\%)<smiles></smiles>

35b (84\%)

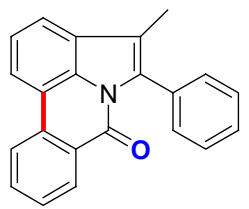

$35 \mathrm{e}(68 \%)$

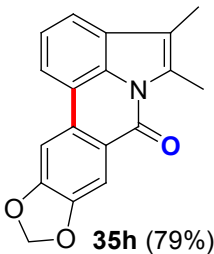

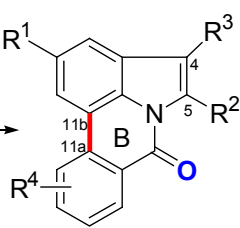

35

21 examples up to $92 \%$ yield<smiles>Cc1c(C)n2c(=O)c3ccccc3c3cc(F)cc1c32</smiles>

35c $(85 \%)$

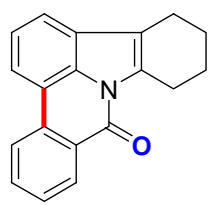

$35 f(90 \%)$<smiles>COC(=O)c1c(C(C)=O)n2c(=O)c3ccccc3c3cccc1c32</smiles>

$35 i$ (NR)

b)

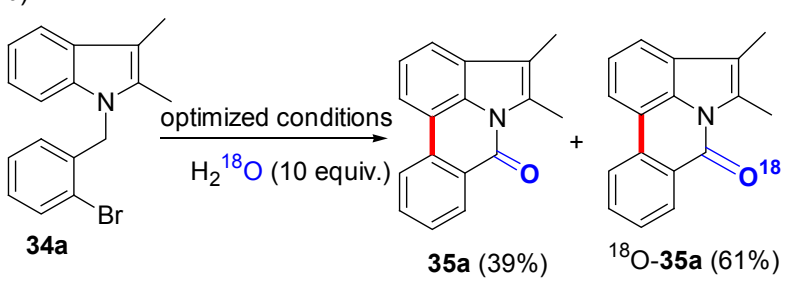

Scheme 8

多学者通过自由基反应构建吡咯并菲啶酮.

1991 年, Lauk 小组 ${ }^{[18]}$ 在合成天然产物 Ungeremine (41)时预先将吲哚啉 7 位用溴活化, 酰化后得到偶联前 体 38. 由于 38 的 $\mathrm{A}$ 环上有两个氢可以参与自由基反应, 因此只能以中等收率得到等比例的 39 和 40 混合物 (Scheme 9). 40 可以经过一系列官能团化反应得到天然 产物 Ungeremine (41). 虽然该方法学的主要目的不是用 于合成 Hippadine (1), 但经过还原、重氮化等繁琐的步 骤脱除硝基可以得到该产物. 上述反应的关键偶联步骤 存在收率不高、反应位点选择性较差等缺陷, 但首次以 自由基反应构建吡咯并菲啶酮母核, 为后续的研究提供 了新的思路.

1998 年, Tsuge 小组 ${ }^{[19]}$ 以 7-溴吲哚 13 为起始原料采 用与 Lauk 小组 ${ }^{[18]}$ 相似的策略合成 Hippadine (1, Scheme 10). 当偶联前体为 43a 时, 虽然不存在反应位点选择性<smiles>[R16]c1ccc2c(c1)OCO2</smiles><smiles>O=C(c1ccc2c(c1)OCO2)N1CCc2cc([N+](=O)[O-])cc(Br)c21</smiles>

38
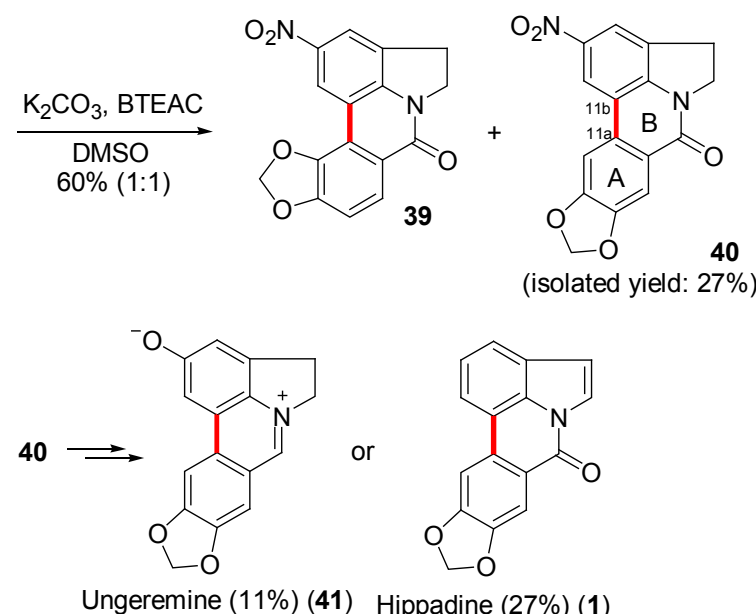

Scheme 9

的问题，但收率依然只有中等 $(52 \%)$; 而当偶联前体为 43b 时，同样由于选择性不佳，收率较低(29\%).
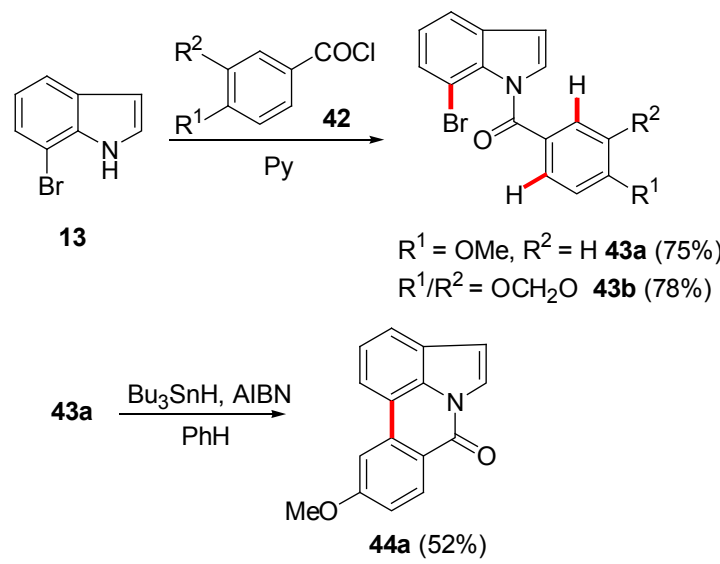

$43 \mathrm{~b}$

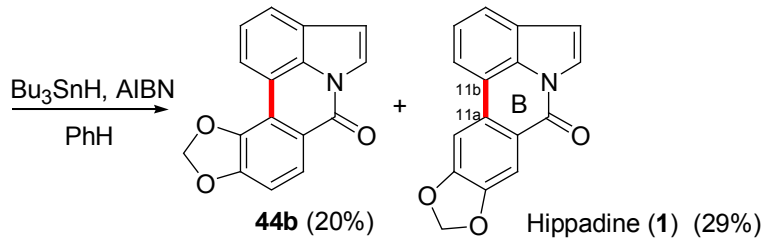

Scheme 10

由上述两个课题组的工作可以看出：通过自由基反 应构建吡咯并菲啶酮母核所用的原料相对容易制备，不 需要对吲哚进行额外的保护, 反应操作也比较简便. 但 是由于反应位点选择性的问题，往往得到不同位点偶联 的混合物，收率和实用性远不如金属催化偶联反应. 因 此，实用价值更高的方法还有待探索. 


\subsection{3 通过其它反应实现}

除上述金属催化偶联和自由基反应以外，也有很多 学者从其它方法入手来构建 $\mathrm{C}_{11 \mathrm{a}}-\mathrm{C}_{11 \mathrm{~b}}$ 键. 1983 年, Frahm 小组 ${ }^{[2]}$ 在报道发现两种全新的生物碱 Pratosine (3)和 Pratorimine (4)的同时, 对其全合成也进行了探索 (Scheme 11). 该方法以吲哚啉 46 和取代邻硝基苯甲酰 氯 45 为起始原料, 经酰化、还原得到伯胺 48 , 伯胺经过 重氮化和关键步骤 Pschorr 偶联得到二氢吡咯并菲啶酮 生物碱 Oxoassoanine (49), 再经过芳构化得到 Pratosine (3), 继续选择性脱除甲基得到 Pratorimine (4). 该路线 操作简捷, 条件温和; 但关键步骤 Pschorr 偶联收率仅 有 $10 \%$, 这显然不能满足大量合成的需要, 还需进一步 改进.
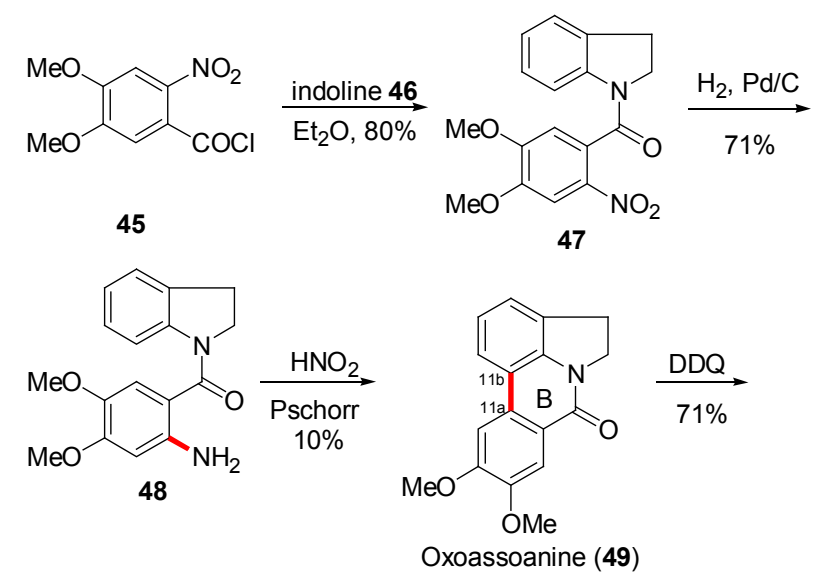

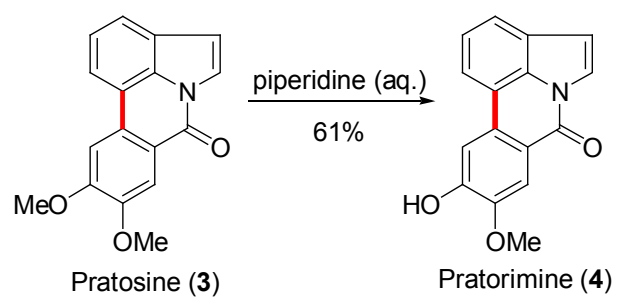

Scheme 11

Ullmann 反应是构建联芳基母核的经典方法 ${ }^{[20]}$, 但 经典的 Ullmann 反应通常需要高温. 1980 年, Ziegler 小 组 ${ }^{[21]}$ 报道采用正丁基锂活化溴苯后, 可以在 $\mathrm{Cu}(\mathrm{I})$ 介导 下在室温中完成 Ullmann 反应. 1999 年, Harrowven 小 组 ${ }^{[22]}$ 将此方法应用到吡咯并菲啶酮的构建中: 通过使 用与前文中 Sakamoto 小组 ${ }^{[8]}$ 类似的底物, 即预先将 $\mathrm{AC}$ 环上的偶联位点 $\mathrm{C}_{11 \mathrm{a}}$ 和 $\mathrm{C}_{11 \mathrm{~b}}$ 采用溴活化, 关键步骤采用 正丁基锂促进的 Ullmann 反应合成 Hippadine (1, Scheme 12). 该路线首次将 Ullmann 反应应用于吡咯并菲啶酮 母核构建中, 总体较为简捷. 而且与其它小组不同的是, 中间体 23 的芐位采用锰酸钡氧化, 操作更加方便, 收率 也比较高. 但关键的 Ullmann 偶联反应收率只有中等, 而且由于用到正丁基锂, 操作比较严格, 官能团耐受程
度不佳，这些不足之处都限制了其应用.
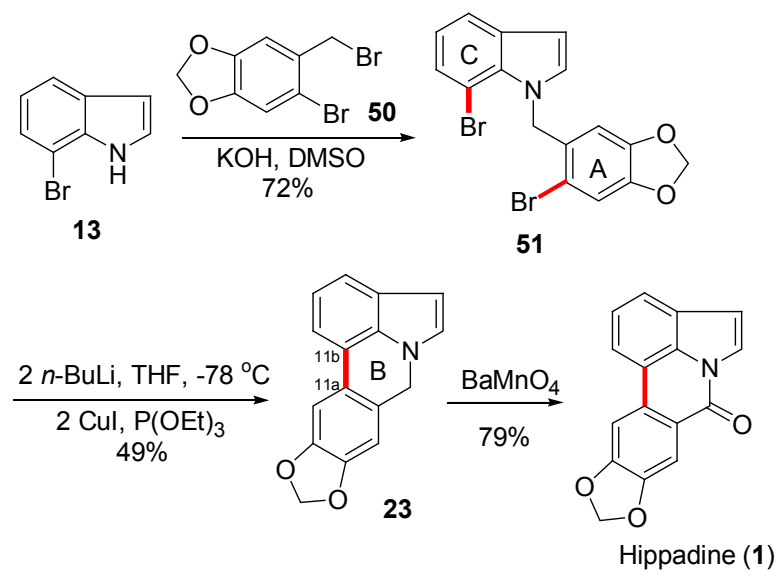

Scheme 12

双(三氟乙酰氧基)碘苯(PIFA)介导的氧化偶联反应 是构建联芳基化合物的常用方法 ${ }^{[23]} .2005$ 年, Kerr 小 组 ${ }^{[24]}$ 报道以简单原料酯 52 和取代苯甲酰胺 53 通过钯催 化得到关键前体吲哚啉衍生物 $\mathbf{5 4 a} / \mathbf{b}$, 继续通过 PIFA 介 导的氧化偶联反应实现吡咯并菲啶酮母核的合成 (Scheme 13). 该方法偶联反应的位点不需预先活化, 操 作较为便捷, 能以很高的收率合成 Hippadine (1). 但中 间体 54a 在进行氧化偶联反应时只能以 $34 \%$ 的收率得到 Oxoassoanine (49), 从而影响了合成 Pratosine (3)的总收 率(Scheme 13). 与前文的方法相比，该方法免除了金属 催化反应脱除 2 位酯基保护的繁琐操作, 改善了自由基 反应区域选择性差、收率低的缺陷，开辟了吡咯并菲啶 酮母核构建的新途径.
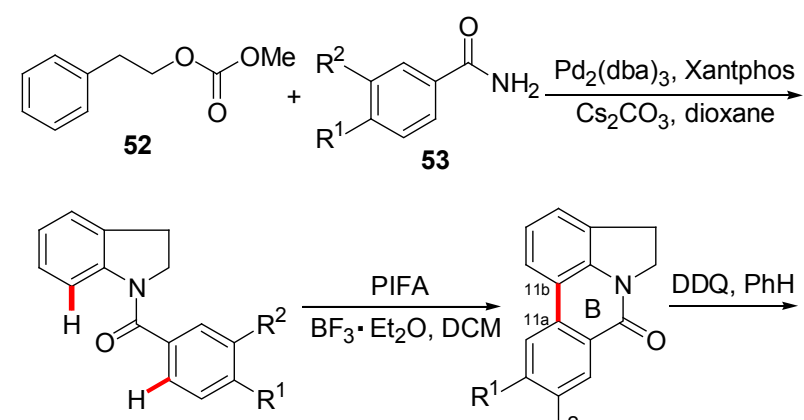

$\mathrm{R}^{1}=\mathrm{R}^{2}=$ OMe $54 \mathrm{a}(97 \%)$

$\mathrm{R}^{1} / \mathrm{R}^{2}=\mathrm{OCH}_{2} \mathrm{O}$ 54b $(85 \%)$

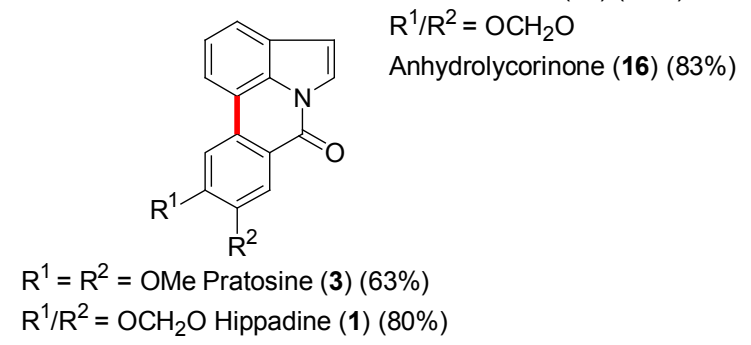

Scheme 13 


\section{2 以构建 $\mathrm{N}_{6}-\mathrm{C}_{7} / \mathrm{C}_{7}-\mathrm{C}_{7 \mathrm{a}}$ 键作为最终环合步骤}

1994 年, Iwao 小组 ${ }^{[25]}$ 采取与之前不同的策略, 即通 过先构建 $\mathrm{C}_{11 \mathrm{a}}-\mathrm{C}_{11 \mathrm{~b}}$ 键, 再构建 $\mathrm{N}_{6}-\mathrm{C}_{7}$ 键合成吡咯并菲 啶酮生物碱(Scheme 14). 该方法以 $N$-Boc 吲哚啉 55 为 起始原料, 经过 7 位锡化反应, 得到偶联底物 56, 与取 代溴苯 $\mathbf{5 7 a} / \mathbf{5 7 b}$ 发生 Stille 偶联得到联苯 $\mathbf{5 8 a} / \mathbf{5 8 b}$. 然后 脱去 Boc 保护, 同时吲哚啉活泼的氨基与醛基发生分子 内环合生成缩醛胺, 缩醛胺被 $\mathrm{Ag}_{2} \mathrm{O}$ 氧化为中间体天然 产物 49/16, 继续芳构化得到目标天然产物 Pratosine (3) 和 Hippadine (1). 同时该小组还报道, 中间体 Anhydroly- corinone (16)可以通过叔丁基锂促进的硼酸 酯化和羟基化, 再经过芳构化得到另外的生物碱 Kalbretorine (2). 该路线采用缩醛胺化环合, 方法比较 新颖, 总收率也令人满意. 此外, 通过中间体 16 可以合 成其它生物碱, 虽然收率还有待提高, 但无疑使该合成 路线的用途更广.
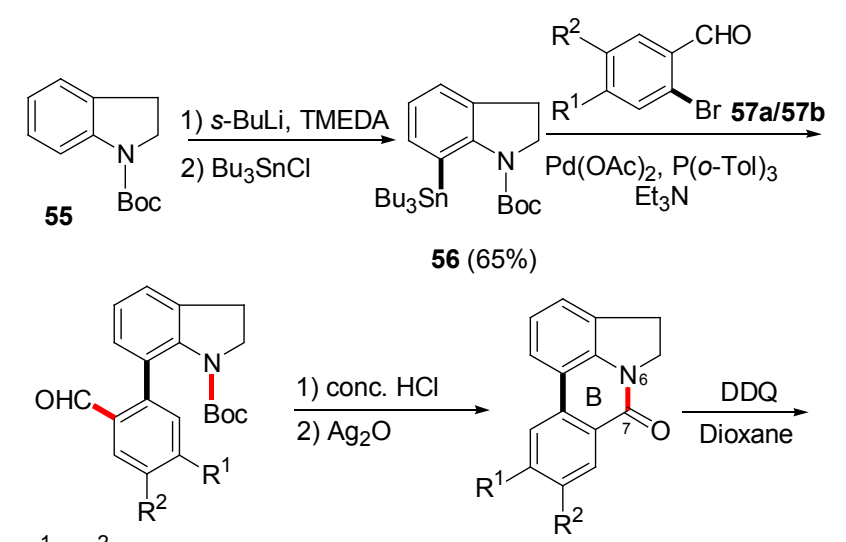

$\mathrm{R}^{1}=\mathrm{R}^{2}=\mathrm{OMe} \mathbf{5 8} \mathrm{a}(65 \%)$

$\mathrm{R}^{1} / \mathrm{R}^{2}=\mathrm{OCH}_{2} \mathrm{O}$ 58b (63\%)

$\mathrm{R}^{1}=\mathrm{R}^{2}=\mathrm{OMe}$

Oxoassoanine (49) (86\%)<smiles>[R]c1ccc2c([R])cc3c(=O)n4cccc4ccc3c2c1</smiles>

$\mathrm{R}^{1} / \mathrm{R}^{2}=\mathrm{OCH}_{2} \mathrm{O}$

Anhydrolycorinone (16) (80\%)

$\mathrm{R}^{1}=\mathrm{R}^{2}=$ OMe Pratosine (3) $(80 \%)$

$\mathrm{R}^{1} / \mathrm{R}^{2}=\mathrm{OCH}_{2} \mathrm{O}$ Hippadine (1) (84\%)

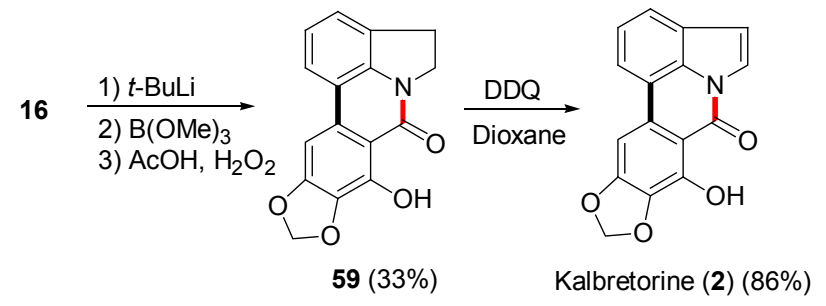

Scheme 14

除 Ullmann 反应外, 格氏反应也是构建碳碳键的经 典方法 ${ }^{[26]} .1996$ 年, Meyers 小组 ${ }^{[27]}$ 将格氏反应应用到吡 咯并菲啶酮的构建方法学中(Scheme 15). 以 $N$-Boc 吲哚
啉 55 为起始原料经过 7 位溴代和保护基转换得到偶联 底物 61. 由于噁唑啉的导向作用, 在 61 和 62 发生的格 氏反应中，反应专一地发生在噁唑啉邻位的甲氧基上， 以较高的收率得到偶联产物 63 . 而化合物 63 中的噁唑 啉部分开环成为羧酸酯, 进一步水解为羧酸, 可以继续 和吲哚啉的氨基环合得到二氢吡咯并菲啶酮 Oxoassoanine (49), 继续芳构化即可得到生物碱 Pratosine (3). Meyers 小组的工作最精彩的地方在于将啞 唑啉的作用发挥到最大：同时作为导向基团和羧酸保护 基，从而一举两得. 该方法学的收率比较高，而且通过 合成不同取代的中间体 63 便可以得到一系列吡咯并菲 啶酩生物碱，因此是一种通用的方法.
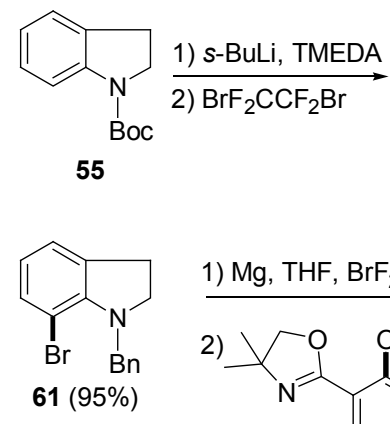<smiles>COc1cc(C2=NC(C)(CC(F)(Br)C(F)(F)Br)CO2)c(-c2cccc3c2N(Cc2ccccc2)CC3)cc1OC</smiles>

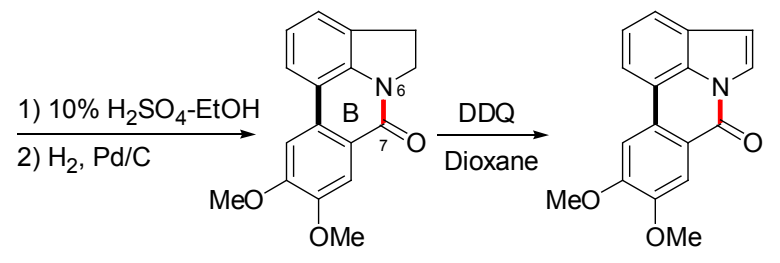

Oxoassoanine (49) (70\%)

\section{Scheme 15}

Bischler-Napieralski 反应(B-N 反应)是构建异喹啉 的经典反应 ${ }^{[28]}$. 但是传统的 B-N 反应通常使用三氯氧磷 作为路易斯酸, 而且在高温下进行, 很多敏感官能团都 不能耐受. 1995 年, Banwell 小组 ${ }^{[29]}$ 对此条件进行改进, 探索出使用三氟乙酸酐/DMAP(摩尔比 5:3)代替三氯氧 磷，在室温下就能以很高的收率得到环合产物. Banwell 小组首次使用 B-N 反应构建 B 环，从而将此方法学应用 于合成一系列吡咯并菲啶酮生物碱(Scheme 16). 该路 线以 7-溴吲哚 13 为起始原料, 与取代苯硼酸 64a/64b 经 过 Suzuki-Miyaura 偶联, 以几乎定量的收率得到关键中 间体 $65 \mathrm{a} / 65 \mathrm{~b}$. 由于该反应的主要目的是合成一系列 4,5-二氢吡咯并菲啶酮生物碱, 因此后续步骤先将吲哚 $65 \mathrm{a} / 65 \mathrm{~b}$ 还原为吲哚啉 $66 \mathrm{a} / 66 \mathrm{~b}$, 再将 $\mathrm{N}$ 上引入甲酯得到 偶联底物 $67 \mathrm{a} / 67 \mathrm{~b}$. 连有甲氧基的底物 $67 \mathrm{a}$ 在探索最优 条件下以良好的收率得到天然产物 Oxoassoanine (49), 
同时伴有少量副产物 68 生成. 而连有亚甲二氧基的底 物 67b 则能以更高的收率 $(88 \%)$ 得到天然产物 Anhydrolycorinone (16), 继续芳构化可得到吡咯并菲啶酮生 物碱 Hippadine (1). Banwell 小组将 B-N 反应改进, 并应 用于吡咯并菲啶酮的构建中, 具有很强的创新性和实用 性：每一步合成路线均经过充分优化，操作简捷，条件 温和; 收率较以往的报道大幅度提高. 该方法是合成吡 咯并菲啶酮类生物碱的代表性方法之一.
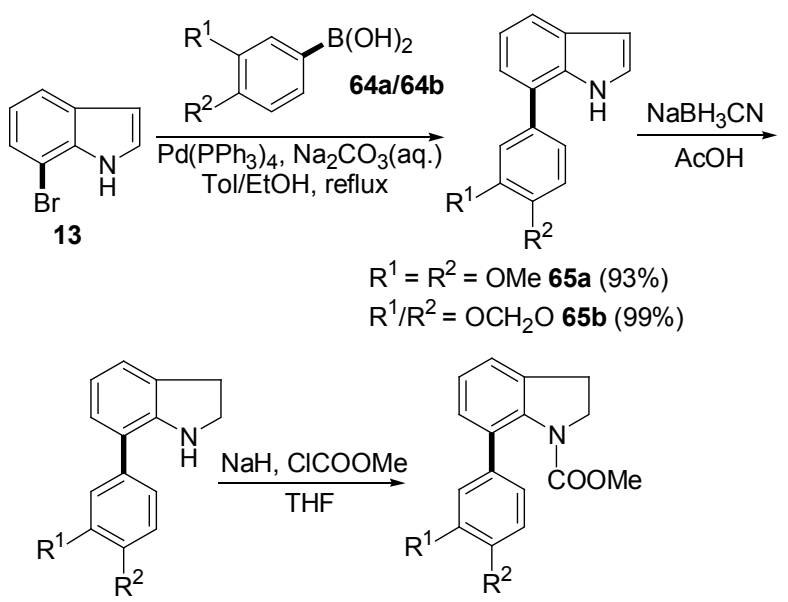

$\mathrm{R}^{1}=\mathrm{R}^{2}=\mathrm{OMe}$ 66a $(77 \%) \quad \mathrm{R}^{1}=\mathrm{R}^{2}=$ OMe 67a $(96 \%)$

$\mathrm{R}^{1} / \mathrm{R}^{2}=\mathrm{OCH}_{2} \mathrm{O} 66 \mathbf{b}(83 \%) \quad \mathrm{R}^{1} / \mathrm{R}^{2}=\mathrm{OCH}_{2} \mathrm{O} 67 \mathrm{~b}(90 \%)$
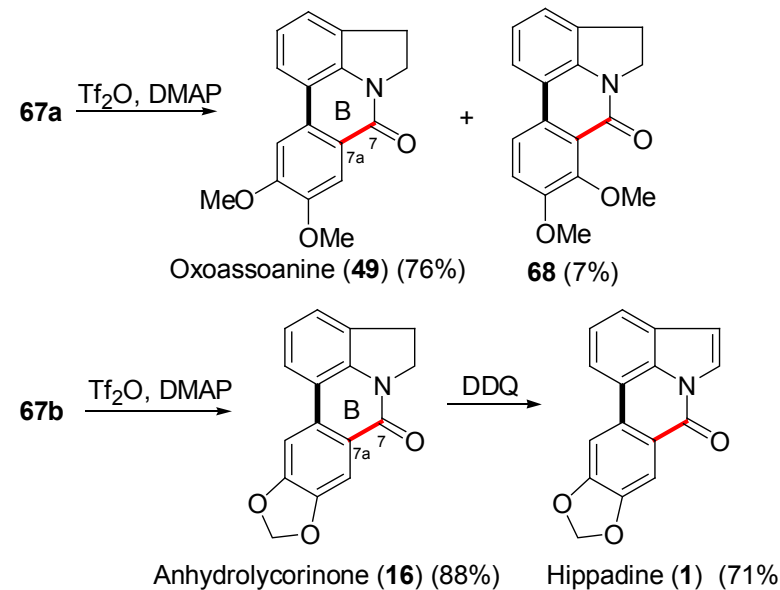

Scheme 16

2004 年, Hiroya 小组 ${ }^{[30]}$ 报道了全新的吲哚合成法: 取代 2-氨基苯乙炔在醋酸铜或三氟醋酸铜催化下, 能以 很高的收率合成吲哚衍生物. Hiroya 小组将此方法学与 上文 Banwell 小组 ${ }^{[29]}$ 的工作结合起来, 用于 Hippadine (1)的合成中(Scheme 17). 该路线以邻碘苯胺 69 起始, 经过 Boc 保护, Sonogashira 反应引入炔基, 再经过碘代 之后与苯硼酸 64b 通过 Suzuki-Miyaura 偶联得到联苯 74. 脱去硅基保护后在铜催化下构建 $\mathrm{D}$ 环得到吲哚 76, 再经过脱 Boc、甲酰化得到关键中间体 77. 77 在三氟乙 酸酐/DMAP 催化下发生 B-N 反应关环得到 Hippadine
(1). 从全合成角度来看, Hiroya 小组的路线关键的 Suzuki 偶联(72\%)和 B-N 反应(84\%)收率都比较高, 但是 关键中间体 77 的合成步骤涉及过多的保护、脱保护步 骤, 比较繁琐, 与上文 Banwell 小组 ${ }^{[29]}$ 的路线相比并没 有显著的优势.
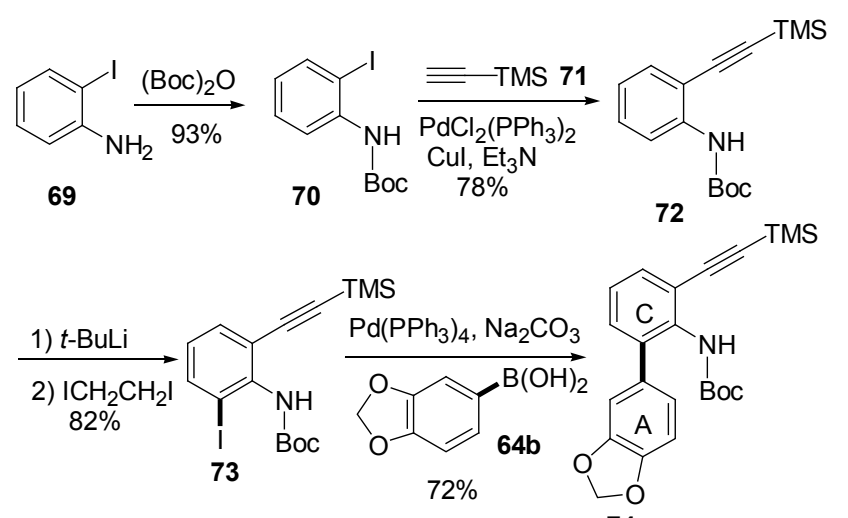

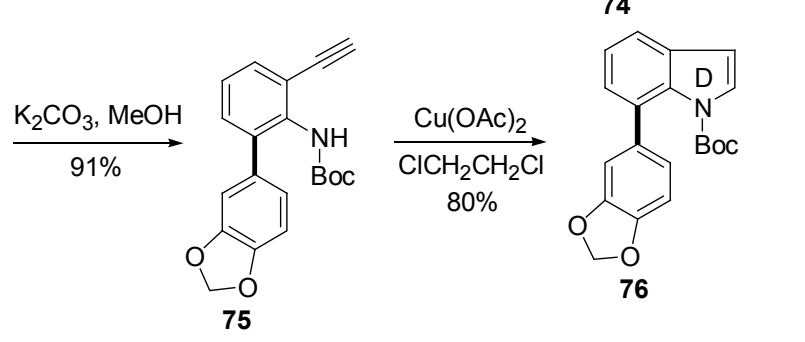

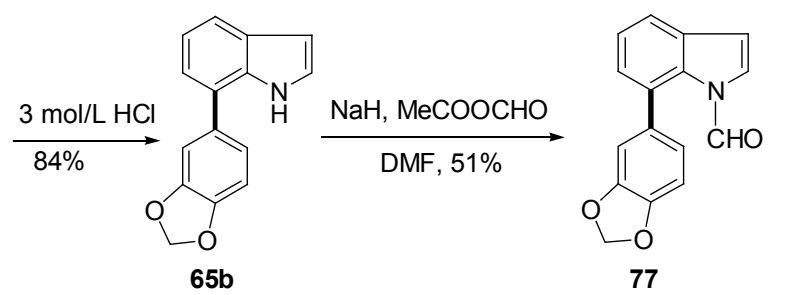

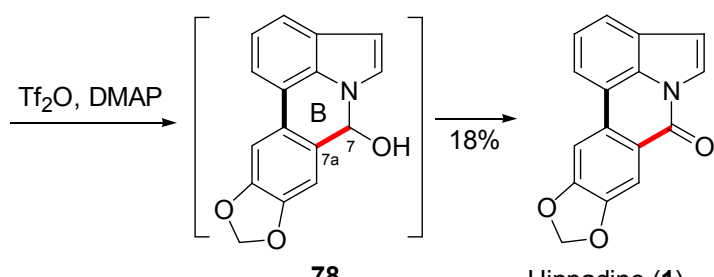

Scheme 17

\section{3 同时构建 $\mathrm{C}_{11 \mathrm{a}}-\mathrm{C}_{11 \mathrm{~b}}$ 键和 $\mathrm{N}_{6}-\mathrm{C}_{7}$ 键}

除上述通过分子内偶联来构建吡咯并菲啶酮母核 外，也有很多学者将偶联位点预先活化，通过分子间偶 联同时构建 $\mathrm{C}_{11 \mathrm{a}}-\mathrm{C}_{11 \mathrm{~b}}$ 键和 $\mathrm{N}_{6}-\mathrm{C}_{7}$ 键. 虽然需要对多个 偶联位点进行预先活化, 但这种方法有很多优点: (1) 几 乎不存在反应位点选择性的问题; (2)可以免去吲哚 2 位 的酯基保护带来的一系列额外的脱除步骤; (3)属于汇聚 式路线，有利于合成效率的提高.

1990 年, Snieckus 小组 ${ }^{[31]}$ 首次通过分子间偶联反应 
实现吡咯并菲啶酮的构建(Scheme 18). Snieckus 小组采 用 $N$-乙酰基吲哚啉 79 为起始原料, 在 7 位碘代之后, 去 保护与取代邻甲酰基苯嗍酸 81 进行 Suzuki-Miyaura 偶 联, 再通过芳构化得到 Hippadine (1). 该路线总体比较 简捷，同时构建 $\mathrm{C}_{11 \mathrm{a}}-\mathrm{C}_{11 \mathrm{~b}}$ 键和 $\mathrm{N}_{6}-\mathrm{C}_{7}$ 键的策略也非常 新颖. 但是吲哚啉 7 位碘代要用到剧毒的 $\mathrm{Tl}\left(\mathrm{OCOCF}_{3}\right)_{3}$, 而且关键步骤 Suzuki-Miyaura 偶联收率只有中等(49\%), 因此还有很大的改进空间.
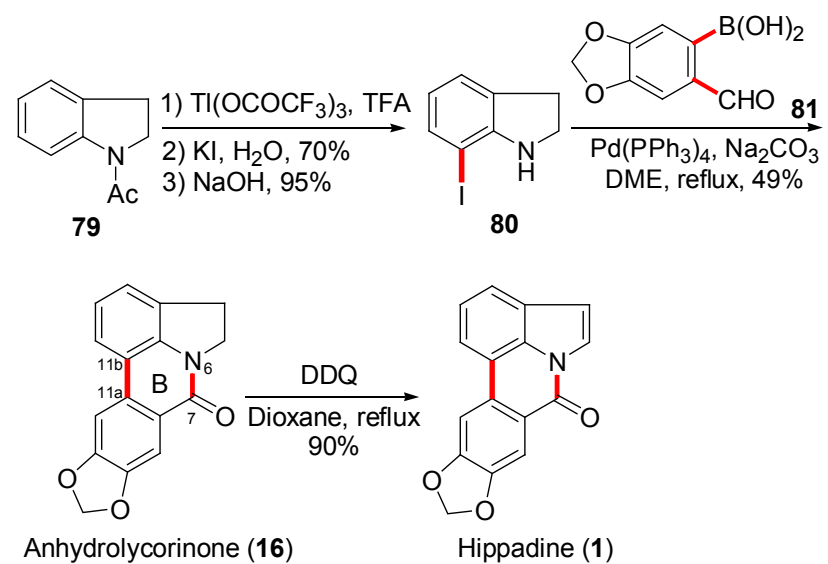

Scheme 18

2003 年, Snieckus 小组 ${ }^{[32]}$ 报道了吲哚衍生物的定向 邻位金属化反应: 即 $N$-酰胺取代吲哚 82 在叔丁基锂作 用下, 选择性在 2 位金属化, 然后加入 $\mathrm{TMSCl}$ 进行硅烷 化以几乎定量收率得到 83 , 继续用仲丁基锂可以选择 性在吲哚 7 位拔氢, 加入嗍酸酯后可以得到吲哚-7-硼酸 酯 84 (Scheme 19). 由于吲哚 $N$-酰基和 2-硅基取代基都 属于易去除基团, 因此该方法学可以用作合成一系列 7取代哚合成子, 在全合成中有广泛应用. Snieckus 小 组将此方法用于 Pratosine (3) 和 Hippadine (1)的合成. 将 84 与取代溴苯 85a 通过 Suzuki-Miyaura 偶联, 能以中等 收率得到偶联产物 86a 和少量的关环产物 Pratosine (4); 而换用 $85 \mathrm{~b}$ 进行偶联, 则能以 $90 \%$ 的收率得到产物 $86 \mathrm{~b}$ 和少量的关环产物 Hippadine (1). 偶联产物 86a/86b 可 以很容易地在碱性条件下关环得到目标天然产物. 该方 法不仅改进了之前用剧毒的 $\mathrm{Tl}\left(\mathrm{OCOCF}_{3}\right)_{3}$ 进行吲哚啉 7 位碘代的严重缺陷，而且在合成 Hippadine (1)时收率非 常高. 遗憾的是, 与之前的方法 ${ }^{[31]}$ 相比, 该反应并不能 很好地实现同时构建 $\mathrm{C}_{11 \mathrm{a}}-\mathrm{C}_{11 \mathrm{~b}}$ 键和 $\mathrm{N}_{6}-\mathrm{C}_{7}$ 键.

2006 年, Tønder 小组 ${ }^{[33]}$ 尝试采用 Kumada、Negishi、 Stille 和 Suzuki-Miyaura 偶联来合成 Hippadine (1). 最终 发现通过 Suzuki-Miyaura 偶联能达到最高的收率 (Scheme 20): 将 7-溴吲哚 13 硼酸酯化之后, 直接加入 取代邻碘苯甲酸甲酯 88, 从而一锅法合成 Hippadine (1). 上述路线的最大亮点在于通过一次性加入较低当
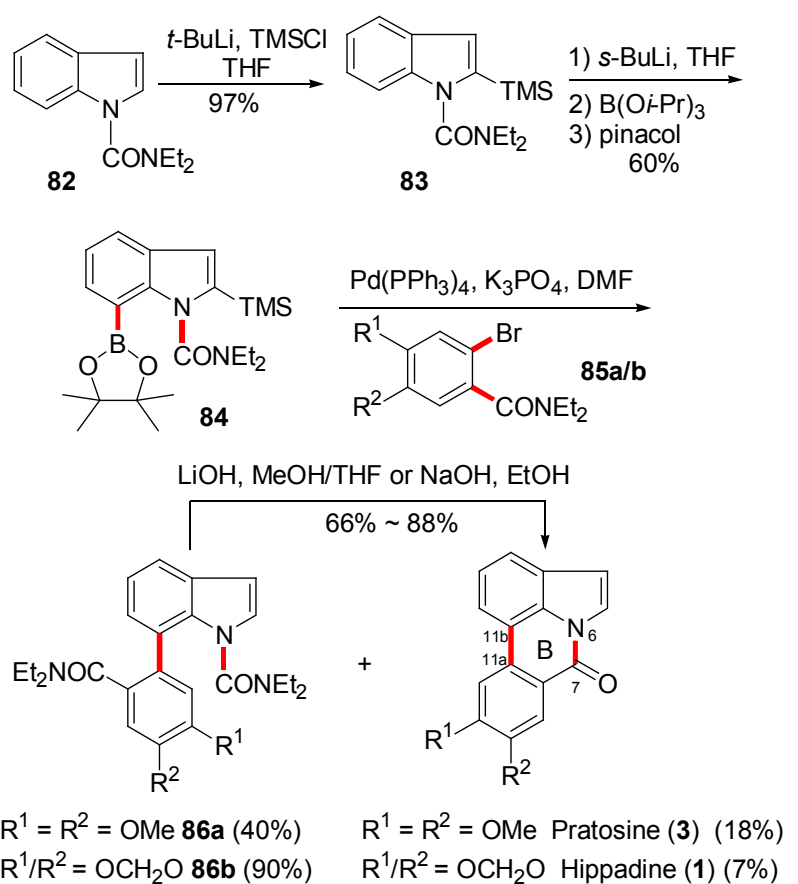

\section{Scheme 19}

量的钯催化剂(5 mol\%), 既合成关键的偶联底物 87 , 又 实现后续的偶联步骤. 此外, 该反应快速，操作简捷;而 且中间不需要多余的分离步骤; 总收率比较理想, 是合 成 Hippadine (1)的经典方法之一.
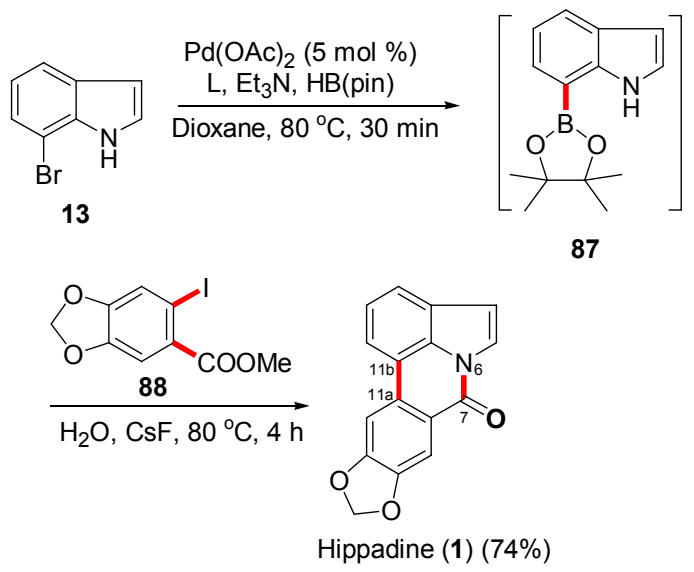

Scheme 20

2010 年, Hartwig 小组 ${ }^{[34]}$ 对前文 Snieckus 小组 ${ }^{[32]}$ 报 道的定向邻位金属化反应进行改进：通过吲哚 $N$-硅基 定位和导向(可能通过铱化的吲哚中间体 90 实现), 在铱 催化下实现吲哚 7-位直接官能团化(Scheme 21). 与 Snieckus 小组的方法相比, 该方法学无需使用叔丁基锂 等危险试剂，反应条件温和，官能团耐受程度更好. Hartwig 小组将此方法学用于吡咯并菲啶酮生物碱的合 成中: 以吲哚 $(89)$ 为起始原料, 在 $\mathrm{N}$ 上引入硅基后, 在 铱催化下发生 7 位嗍酸酯化, 得到的与 Tønder 小组相同 
的中间体 87 , 直接向反应液中加入取代邻溴苯甲酸甲 酯 88 和钯催化剂, 一锅法以 48\%总收率得到 Hippadine (1). 该路线与前文 Tønder 小组 ${ }^{[33]}$ 的路线相似, 虽然总 收率不及前者, 但起始原料为吲哚, 显然更加廉价易得, 这在合成吲哚环上有取代基的天然产物时优势更加明 显. 该方法也是合成 Hippadine (1)的优良方法之一.

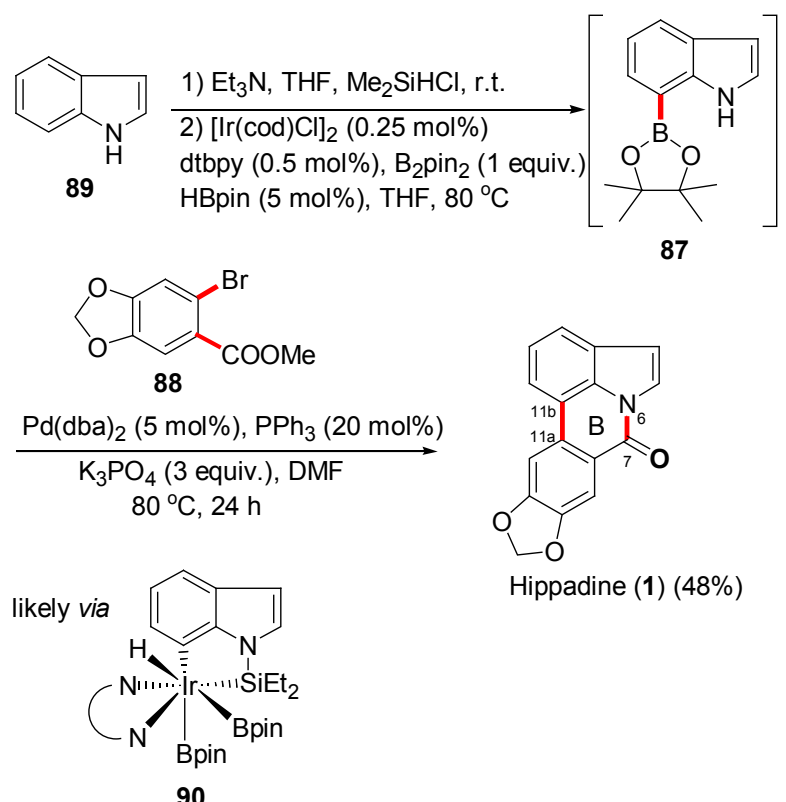

Scheme 21

2013 年, Banwell 小组 ${ }^{[35]}$ 在上文 Hartwig 小组 ${ }^{[34]}$ 方 法学和全合成探索的基础上, 以取代吲哚 91 起始, 关键 步骤同样采用 Suzuki-Miyaura 偶联, 最终合成 Lycoranine A (7)、Lycoranine B (8) 以及 2-methoxypratosine (93)三种天然生物碱(Scheme 22). 与 Hartwig 小组不同的是, 该路线采取分步进行, 并改善了 SuzukiMiyaura 偶联的收率, 使得总收率显著提高. 同时该路 线也是目前合成 Lycoranine A (7)和 Lycoranine B (8) 的 唯一报道.

上述 Snieckus、Tønder 和 Banwell 小组的关键偶联 步骤都是通过 Suzuki-Miyaura 偶联实现, 上述合成方法 底物易于制备和修饰，反应路线比较简捷、易于实现， 收率比较理想, 代表了通过分子间偶联构建吡咯并菲啶 酮的主流方法.

\section{2 以构建 CD 环为关键步骤}

吡咯并菲啶酮的 $\mathrm{CD}$ 环为吲哚环, 而吲哚来源广泛, 比较易得, 因此从构建 CD 环入手的方法学报道比较少. 此外，该策略与前述的方法相比并没有明显优势，因此 在本文只作简单讨论.

1996 年, Lobo 小组 ${ }^{[36]}$ 报道了一种构建吡咯并菲啶

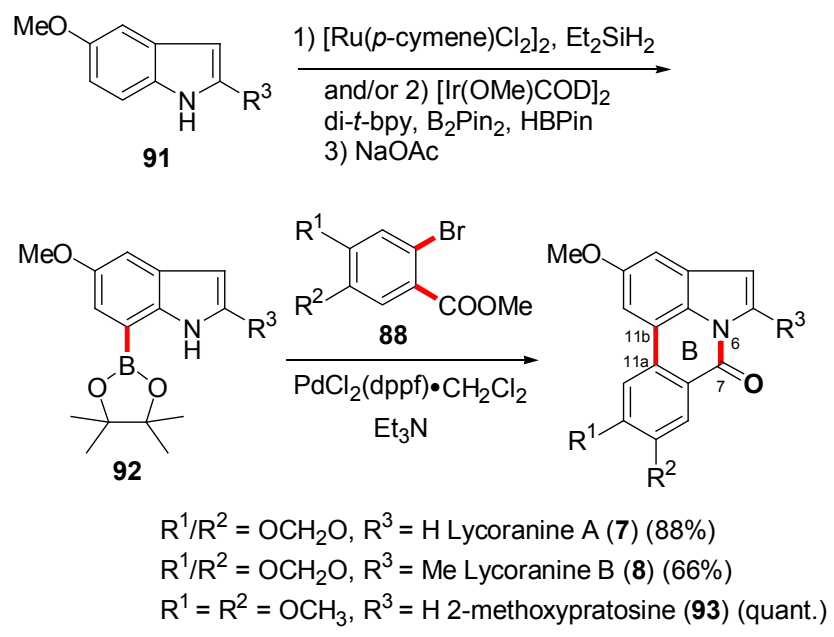

Scheme 22

酮母核的全新策略: 即先构建 $\mathrm{ABC}$ 环，最后构建 D 环 (Scheme 23). 以取代邻溴苯甲酰氯 94 和 $N$-苯基羟胺 95 为起始原料, 在三氟化嗍乙醚的作用下缩合得到氟嗍酸 盐 96, 继续在紫外下发生分子内关环得到具有 $\mathrm{ABC}$ 环 结构的菲啶酮 97.97 水解后游离出羟基，和丙炔酸甲酯 99 发生 Michael 加成得到关键中间体 100. 中间体 $\mathbf{1 0 0}$ 加热发生一系列分子内 3,3-迁移、关环得到吡咯并菲啶 酮 $101(33 \%)$, 同时会有少部分 (17\%) 脱酯基得到 Pratosine (3). 同时，中间体 100 在热解下会有其他副产 物产生，比如 $\mathrm{N}-\mathrm{O}$ 键断裂产物 102 (25\%)或 1,3-迁移产 物 103 (15\%), 而 103 在此条件下无法继续关环成为吡 咯并菲啶酮. 可以看出，上述路线的设计思路非常独特， 采用简单原料可以迅速构建具备 $\mathrm{ABC}$ 环的菲啶酤 $\mathbf{9 8}$, 通过 Michael 加成引入 D 环所需的双键并最终通过分子 内 3,3-迁移和关环得构建 D 环. 但是最后一步热解副产 物较多, 主产物 101 和 Pratosine (3) 的收率都不令人满 意，因此该方法并不具备很强的实用价值，只能提供一 种构建吡咯并菲啶酮的新思路.

Diels-Alder 反应是全合成中应用最广泛的反应之 — ${ }^{[37]}$ ，但是在吡咯并菲啶酮的构建中却很少见. 2000 年, Boger 小组 ${ }^{[38]}$ 报道了采用分子内 Diels-Alder 反应同时构 建 $\mathrm{BC}$ 环 (Scheme 24). 首先以取代邻溴苯甲醛 57b 为起 始原料, 经过一系列官能团转换得到邻甲酰基苯甲酸甲 酯 107, 经 Wittig 反应、甲酯水解得到关键中间体 110 . 另外一个偶联组分的合成是以四嗪 111 起始，与炔基胺 112 单胺化反应得到 113. 有趣的是, 113 在 Boc 保护时 发生分子内 $[4+2]$ 环合得到 114, 脱去 Boc 保护得到关 键中间体 115.115 与前面合成的中间体 110 缩合得到偶 联前体 116. 116 在高温下发生分子内 Diels-Alder 反应同 时构建 $\mathrm{BC}$ 环得到吡咯并菲啶酮 117. 再经过脱甲硫基 和芳构化得到天然产物 Hippadine (1). Boger 小组的合成 策略非常新颖, 关键的两步分子内 $[4+2]$ 环合收率均达 
<smiles>CCOC(=O)c1cc(Br)ccc1NO</smiles><smiles>COc1cc(Br)c(/C(=N\c2ccccc2)O[Pb](F)(F)F)cc1[O+]</smiles><smiles>COc1cc2c3ccccc3n(O)c2cc1OC</smiles>

97

98

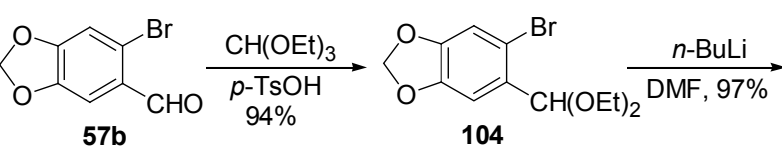

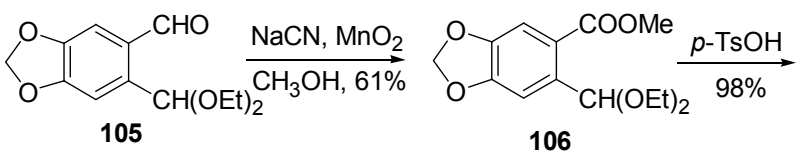<smiles>[R]OC(=O)c1cc2c(cc1/C=C\OC)OCO2</smiles>

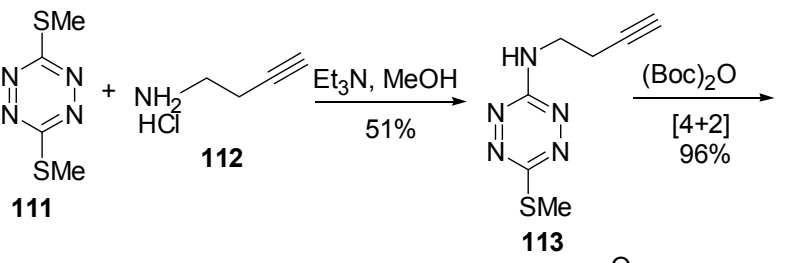

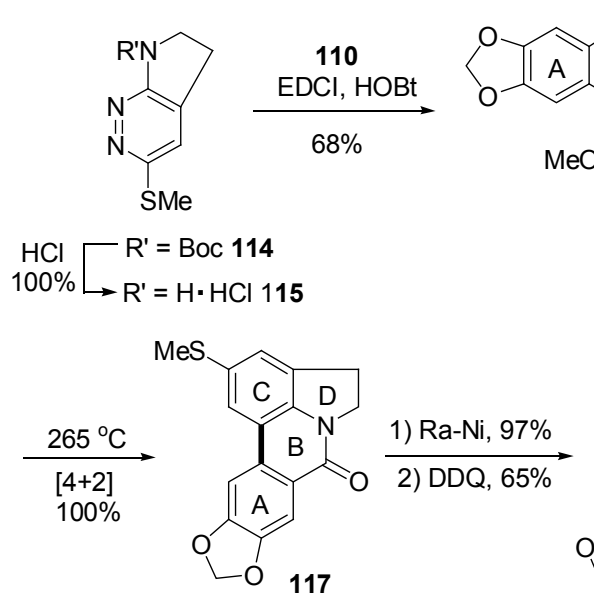

Scheme 24

\section{References}

[1] Martin, S. F. In The Alkaloids, Ed.: Brossi, A., Academic Press, New York, 1987, Vol. 30, Chapter 3, p. 251.

[2] (a) Pigni, N. B.; Ríos-Ruiz, S.; Martínez-Francés, V.; Nair, J. J.; Viladomat, F.; Codina, C.; Bastida, J. J. Nat. Prod. 2012, 75, 1643.

(b) Wang, L.; Zhang, X.-Q.; Yin, Z.-Q.; Wang, Y.; Ye, W.-C. Chem. Pharm. Bull. 2009, 57, 610.

(c) Ramadan, M. A.; Kamel, M. S.; Ohtani, K.; Kasai, R.; Yamasaki, K. Phytochemistry 2000, 54, 891.

(d) Ghosal, S.; Lochan, R.; Kumar, A. Y.; Srivastava, R. S. Phytochemistry 1985, 24, 1825.

(e) Ghosal, S.; Saini, K. S.; Frahm, A. W. Phytochemistry 1983, 22, 2305.

(f) Ghosal, S.; Rao, P. H.; Jaiswal, D. K.; Kumar, Y.; Frahm, A. W. Phytochemisrty 1981, 20, 2003.

[3] (a) Min, B. S.; Gao, J. J.; Nakamura, N.; Kim, Y. H.; Hattori, M. Chem. Pharm. Bull. 2001, 49, 1217.

(b) Ali, A. A.; Mesbah, M. K.; Frahm, A. W. Planta Med. 1981, 43, 
407.

(c) Zee-Cheng, R. K.-Y.; Yan, S.-J.; Cheng, C. C. J. Med. Chem 1978, 21, 199.

[4] Chattopadhyay, S.; Chattopadhyay, U.; Mathur, P. P.; Saini, K. S.; Ghosal, S. Planta Med. 1983, 49, 252.

[5] For selected reviews on transition-metal catalyzed functionalization of arenes, see:

(a) Johansson Seechurn, C. C. C.; Kitching, M. O.; Colacot, T. J.; Snieckus, V. Angew. Chem. Int. Ed. 2012, 51, 5062.

(b) Kuhl, N.; Hopkinson, M. N.; Wencel-Delord, J.; Glorius, F. Angew. Chem., Int. Ed. 2012, 51, 10236.

(c) Neufeldt, S. R.; Sanford, M. S. Acc. Chem. Res. 2012 , 45, 936.

(d) Bras, J. L.; Muzart, J. Chem. Rev. 2011, 111, 1170.

(e) Sun, C.-L.; Li, B.-J.; Shi, Z.-J. Chem. Rev. 2011, 111, 1293.

(f) Liu, C.; Zhang, H.; Shi, W.; Lei, A. Chem. Rev. 2011, 111, 1780.

(g) Roger, J.; Gottumukkala, A. L.; Doucet, H. ChemCatChem 2010, 2, 20.

(h) Johansson, C. C. C.; Colacot, T. J. Angew. Chem., Int. Ed. 2010, $49,676$.

(i) Chen, X.; Engle, K. M.; Wang, D.-H.; Yu, J.-Q. Angew. Chem., Int. Ed. 2009, 48, 5094.

[6] For a selected review on palladium-catalyzed intramolecular cylization of heteroarenes, see:

(a) You, S.-L.; Xia, J.-B. Top. Curr. Chem. 2010, 292, 165.

For selected examples, see:

(b) Zhao, L.; Li, Z.; Chang, L.; Xu, J.; Yao, H.; Wu, X. Org. Lett. 2012, 14, 2066.

(c) Piou, T.; Neuville, L.; Zhu, J. Tetrahedron 2013, 69, 4415.

(d) Wu, K.-J.; Dai, L.-X.; You, S.-L. Org. Lett. 2012, 14, 3772.

(e) Lv, J.; Liu, Q.; Tang, J.; Perdih, F.; Kranjc, K. Tetrahedron Lett. 2012, 53, 5248 .

(f) Yamuna, E.; Zeller, M.; Prasad, K. J. R. Tetrahedron Lett. 2011, 52,6030 .

(g) Wang, C.; Piel, I.; Glorius, F. J. Am. Chem. Soc. 2009, 131, 4194.

(h) Rudolph, A.; Rackelmann, N.; Turcotte-Savard, M.-O.; Lautens, M. J. Org. Chem. 2009, 74, 289.

(i) Zhang, H.; Ferreira, E. M.; Stoltz, B. M. Angew. Chem., Int. Ed. 2004, 43, 6144.

(j) Huang, Q.; Fazio, A.; Dai, G.; Campo, M. A.; Larock, R. C. J. Am. Chem. Soc. 2004, 126, 7460.

(k) Ferreira, E. M.; Stoltz, B. M. J. Am. Chem. Soc. 2003, 125, 9578.

(1) Campo, M. A.; Huang, Q.; Yao, T.; Tian, Q.; Larock, R. C. J. Am. Chem. Soc. 2003, 125, 11506.

[7] Grigg, R.; Teasdale, A.; Sridharan, V. Tetrahedron Lett. 1991, 32, 3859 .

[8] Sakamoto, T.; Yasuhara, A.; Kondo, Y.; Yamanaka, H. Heterocycles 1993, 36, 2597.

[9] Knölker, H.-J.; Filali, S. Synlett 2003, 1752.

[10] For selected examples, see:

(a) Pinto, A. C.; Silva, F. S. Q.; Silva, R. B. Tetrahedron Lett. 1994, 35, 8923 .

(b) Garden, S. J.; Silva, R. B.; Pinto, A. C. Tetrahedron 2002, 58, 8399 .

[11] Torres, J. C.; Pinto, A. C.; Garden, S. J. Tetrahedron 2004, 60, 9889.

[12] Miki, Y.; Shirokoshi, H.; Matsushita, K. Tetrahedron Lett. 1999, 40, 4347.
[13] Umemoto, H.; Dohshita, M.; Hamamoto, H.; Miki, Y. Heterocycles 2011, 83, 1111.

[14] Miki, Y.; Umemoto, H.; Dohshita, M.; Hamamoto, H. Tetrahedron Lett. 2012, 53, 1924.

[15] Cong, W.; Zhao, L.; Wu, X.; Xu, J.; Yao, H. Tetrahedron 2014, 70, 312 .

[16] For selected examples, see:

(a) Xie, Y.; Yang, Y.; Huang, L.; Zhang, X.; Zhang, Y. Org. Lett. 2012, 14, 1238.

(b) Wang, Z.-Q.; Zhang, W.-W.; Gong, L.-B.; Tang, R.-Y.; Yang, X.-H.; Liu, Y.; Li, J.-H. Angew. Chem., Int. Ed. 2011, 50, 8968.

[17] Rosen, B. M.; Percec, V. Chem. Rev. 2009, 109, 5069.

[18] Lauk, U.; Dürst, D.; Fischer, W. Tetrahedron Lett. 1991, 32, 65.

[19] Tsuge, O.; Hatta, T.; Tsuchiyama, H. Chem. Lett. 1998, 155.

[20] Sperotto, E.; van Klink, G. P.; van Koten, G.; de Vries, J. G. Dalton Trans. 2010, 39, 10338.

[21] Ziegler, F. E.; Chliwner, I.; Fowler, K. W.; Kanfer, S. J.; Kuo, S. J.; Sinha, N. D. J. Am. Chem. Soc. 1980, 102, 790.

[22] Harrowven, D. C.; Lai, D.; Lucas, M. C. Synthesis 1999, 1300.

[23] Hiroya, K.; Itoh, S.; Sakamoto, T. J. Org. Chem. 2004, 69, 1126.

[24] Ganton, M. D.; Kerr, M. A. Org. Lett. 2005, 7, 4777.

[25] Iwao, M.; Takehara, H.; Obta, S.; Watanabe, M. Heterocycles 1994, $38,1717$.

[26] Harutyunyan, S. R.; den Hartog, T.; Geurts, K.; Minnaard, A. J.; Feringa, B. L. Chem. Rev. 2008, 108, 2824.

[27] Hutchings, R. H.; Meyers, A. I. J. Org. Chem. 1996, 61, 1004.

[28] For selected examples, see:

(a) Fodor, G.; Nagubandi, S. Tetrahedron 1980, 36, 1279.

(b) Medley, J. W.; Movassaghi, M. Org. Lett. 2013, 15, 3614.

(c) Su, B.; Chen, F.; Wang, Q. J. Org. Chem. 2013, 78, 2775.

(d) Adachi, S.; Onozuka, M.; Yoshida, Y.; Ide, M.; Saikawa, Y.; Nakata, M. Org. Lett. 2014, 16, 358.

[29] Banwell, M. G.; Bissett, B. D.; Busato, S.; Cowden, C. J.; Hockless, D. C. R.; Holman, J. W.; Read, R. W.; Wu, A. W. J. Chem. Soc., Chem. Commun. 1995, 2551.

[30] Hiroya, K.; Itoh, S.; Sakamoto, T. J. Org. Chem. 2004, 69, 1126.

[31] Siddiqui, M. A.; Snieckus, V. Tetrahedron Lett. 1990, 31, 1523.

[32] Hartung, C. G.; Fecher, A.; Chapell, B.; Snieckus, V. Org. Lett. 2003, 5, 1899.

[33] Mentzel, U. V.; Tanner, D.; Tønder, J. E. J. Org. Chem. 2006, 71, 5807.

[34] Robbins, D. W.; Boebel, T. A.; Hartwig, J. F. J. Am. Chem. Soc. 2010, 132, 4068.

[35] Kim, H. S.; Banwell, M. G.; Willis, A. C. J. Org. Chem. 2013, 78, 5103.

[36] Pereira, M. M. A.; Prabhakar, S.; Lobo, A. M. J. Nat. Prod. 1996, 59, 744.

[37] For selected examples, see:

(a) Jia, Z.-J.; Jiang, H.; Li, J.-L.; Gschwend, B.; Li, Q.-Z.; Yin, X.; Grouleff, J.; Chen, Y.-C.; Jørgensen, K. A. J. Am. Chem. Soc. 2011, 133, 5053.

(b) Liu, Y.; Nappi, M.; Arceo, E.; Vera, S.; Melchiorre, P. J. Am. Chem. Soc. 2011, 133, 15212.

(c) Lin, S.; Ischay, M. A.; Fry, C. G.; Yoon, T. P. J. Am. Chem. Soc. 2011, 133, 19350. For selected reviews, see:

(d) Jiang, X.; Wang, R. Chem. Rev. 2013, 113, 5515 .

(e) Funel, J. A.; Abele, S. Angew. Chem., Int. Ed. 2013, 52, 3822.

[38] Boger, D. L.; Wolkenberg, S. E. J. Org. Chem. 2000, 65, 9120. 